OPEN ACCESS

Edited by:

Haoyu Chen,

Shantou University \& the Chinese

University of Hong Kong, China

Reviewed by:

Andrea Govetto,

ASST Fatebenefratelli Sacco, Italy

Pratyusha Ganne,

All India Institute of Medical Sciences,

Mangalagiri, India

William Foster,

Temple University, United States

*Correspondence:

Hejiang Ye

yehej@163.com

Specialty section:

This article was submitted to

Ophthalmology,

a section of the journal

Frontiers in Medicine

Received: 04 July 2021 Accepted: 24 November 2021 Published: 23 December 2021

Citation:

Zhou Q, Feng $H, L v H, F u Z$, Xue Y

and Ye $H$ (2021) Vitrectomy $v s$. Spontaneous Closure for Traumatic Macular Hole: A Systematic Review and Meta-Analysis.

Front. Med. 8:735968.

doi: 10.3389/fmed.2021.735968

\section{Vitrectomy vs. Spontaneous Closure for Traumatic Macular Hole: A Systematic Review and Meta-Analysis}

\author{
Qi Zhou ${ }^{1,2}$, Haoyue Feng ${ }^{1,2}$, Hongbin $L^{3}{ }^{3}$, Zhongmei Fu ${ }^{1,2}$, Yuyu Xue ${ }^{1,2}$ and Hejiang $\mathrm{Ye}^{2 *}$ \\ 'Eye School, Chengdu University of Traditional Chinese Medicine, Chengdu, China, ${ }^{2}$ Department of Ophthalmology, \\ Affiliated Hospital of Chengdu University of Traditional Chinese Medicine, Chengdu, China, ${ }^{3}$ Department of Ophthalmology, \\ Affiliated Hospital of Southwest Medical University, Luzhou, China
}

Objective: This systematic review and meta-analysis aimed to determine the traumatic macular hole $(\mathrm{TMH})$ closure rate and visual acuity (VA) improvement rate by comparing two treatment methods for $\mathrm{TMH}$ : vitrectomy and observation for spontaneous closure.

Methods: PubMed, Cochrane, Web of Science Library, Embase, CNKI, Wanfang, VIP, and Sino Med were systematically searched from their inception to June 10, 2021. Studies in the surgery group $(n=32)$ and studies in the observation group $(n=12)$ were meta-analyzed. The primary outcomes were the TMH closure and VA improvement rates in the surgery and observation groups. The secondary outcomes were best-corrected visual acuity (BCVA) improvement in the surgery group. Stata software (version 15.1) was used for the analyses.

Results: Thirty-six studies that included 1,009 eyes were selected for this meta-analysis, among which 33 were retrospective studies and 3 were prospective studies. The meta-analysis showed that the random-model pooled event rate for $\mathrm{TMH}$ closure was 0.37 (95\% confidence interval [Cl], 0.26-0.48) in the observation group, while it was 0.9 (95\% Cl, 0.85-0.94) in the surgery group. The fixed-model pooled event rate for VA improvement was $0.39(95 \% \mathrm{Cl}, 0.33-0.45)$ in the observation group, while the random-model pooled event rate of VA improvement for the surgery group was 0.72 (95\% Cl, 0.63-0.80). The pooled event rate for BCVA improvement in the surgery group was $0.39(95 \% \mathrm{Cl}, 0.33-0.46)$.

Conclusions: This meta-analysis suggests that TMH hole closure and VA improvement rates in the surgery group were significantly higher than those in the observation group. Vitrectomy is an effective method for treating $\mathrm{TMH}$. However, further randomized controlled trials (RCTs) are required to evaluate the efficacy and safety of surgery and observation for $\mathrm{TMH}$ treatment.

Systematic Review Registration: https://www.crd.york.ac.uk/PROSPERO/\# recordDetails, identifier: CRD42021276684.

Keywords: traumatic macular hole, closure rate, visual acuity improvement, vitrectomy, spontaneous closure, meta-analysis 


\section{INTRODUCTION}

A macular hole $(\mathrm{MH})$ is a defined as a full-thickness defect of the neuroretina in the macular foveal area. Traumatic macular holes (TMH) represent approximately $10 \%$ of MHs and may result in permanent significant vision loss (1). TMH is often found in young men, as the condition it is frequently associated with sport- and work-related accidents (2). The functional outcomes are often unclear because of the accompanying traumainduced retinal pathologies, such as vitreous hemorrhage, retinal detachment, retinal hemorrhage, choroidal fracture, subretinal choroidal neovascularization, and fibrosis.

However, the posttraumatic approach is controversial. To date, no clinical guidelines have been established for this visionthreatening disease. Treatment includes vitrectomy surgery and observation, as well as spontaneous closure (1). Vitrectomy has been reported to improve anatomical and visual outcomes in eyes with $\mathrm{TMH}(3,4)$. Currently, surgical techniques include removing the posterior vitreous cortex and epiretinal membranes, with or without internal limiting membrane (ILM) peeling, and intraocular gas or silicone oil tamponade. Various adjuvant therapies, including transforming growth factor-beta (TGF- $\beta$ ), biological tissue adhesives, and platelet concentrate, have been investigated with varying degrees of success (5, 6). However, there are many unanswered questions about the necessity of surgery because spontaneous hole closure has been commonly reported (7). Many studies have reported that spontaneous closure usually occurs between 1 and 6 months after the trauma incident $(8,9)$. While a number of studies have discussed the anatomical and visual outcomes of surgery and observation on $\mathrm{TMH}$, a previous systemic review and metaanalysis, which included only 10 studies, lacked sufficient detail (10). Therefore, in this systematic review and meta-analysis, we systematically and statistically determined the improvement rates with TMH closure and visual acuity (VA) by comparing the two methods of treating TMH.

\section{MATERIALS AND METHODS}

This systematic review and meta-analysis was performed according to the Meta-analyses Of Observational Studies in Epidemiology (MOOSE) guidelines (11). Ethical approval was not necessary for the study as it used published data. Four databases in English, including PubMed, Cochrane, Web of Science Library, and Embase, and four databases in Chinese, including CNKI, Wanfang, VIP, and Sino Med, were searched from their inception to June 10, 2021. Google Scholar and Baidu Scholar were also searched to find studies missing in those databases. A manual search was conducted to identify published studies. In the case of unpublished studies, the database was searched for their abstracts, and their authors were also contacted. EndNote was used to merge retrieved citations and eliminate duplications.

Two independent researchers (QZ and HYF) separately assessed the eligibility, extracted the data, and assessed the quality of the included studies, and a third author (HJY) determined the final criteria for any inconsistencies.

\section{Search Strategy}

The search strategy included the following search terms: "retinal perforations," "retinal hole," "retinal tear," "retinal break," "macular hole," "traumatic macular hole," "vitrectomy," "pars plana vitrectomy," "surgical management," "observation," "treatment," and "spontaneous closure." The search terms are shown in the Supplementary Material.

\section{Inclusion Criteria}

Articles were included if they (1) included studies on patients with $\mathrm{TMH}$; (2) used closure rate and VA improvement rate as the treatment endpoints; (3) provided clinical statistics on age, sex, best-corrected visual acuity (BCVA) expressed in the logarithm of the minimal angle of resolution (logMAR), MH size, follow-up data, operation, interval from injury to surgery, TMH closure rate, and VA improvement rate; and (4) were published in Chinese or English full text.

\section{Exclusion Criteria}

Articles were excluded if they (1) reported duplicated or overlapping data; (2) were designed as "reviews," "case reports," "letters" or "conference articles" with no data to extract; (3) focused on patients diagnosed with idiopathic $\mathrm{MH}$, myopic $\mathrm{MH}$, or TMH with retinal detachment; and (4) were not published in Chinese or English full text.

\section{Data Extraction}

According to the inclusion and exclusion criteria, two researchers (QZ and HYF) independently read the full text of the articles and selected the qualified studies. The following information was extracted from eligible studies: first author and publication year, study design, country, follow-up time, sex, number of participants, BCVA logMAR before and after TMH closure, size of TMH $(\mu \mathrm{m})$, closure rate, and VA improvement rate. For the surgery group patients, data on the interval from the injury to the surgery were extracted. For the observation group patients, data on the time of hole closure were extracted.

\section{Quality Assessment}

To accurately evaluate the methodological quality of eligible studies, two researchers independently used the NewcastleOttawa Scale, which is a nine-point system including participant selection ( $0-4$ points), comparability ( $0-2$ points), and exposure (0-3 points) (12). Scores of 0-3, 4-6, and 7-9 points were regarded as low, moderate, and high quality, respectively. All included studies were identified to be of moderate or high methodological quality (Supplementary Material).

\section{Statistical Analysis}

The primary outcomes were TMH closure rate and VA improvement rate in both groups. BCVA improvement in the surgery group was defined as a secondary outcome.

Continuous data are presented as mean \pm standard deviation. The descriptive statistics (BCVA logMAR improvement, TMH closure, and VA improvement rate) were analyzed with a $95 \%$ confidence interval (CI). The Cochran $\mathrm{Q}$ test and $I^{2}$ statistic were used to identify heterogeneity among the studies. When there was 


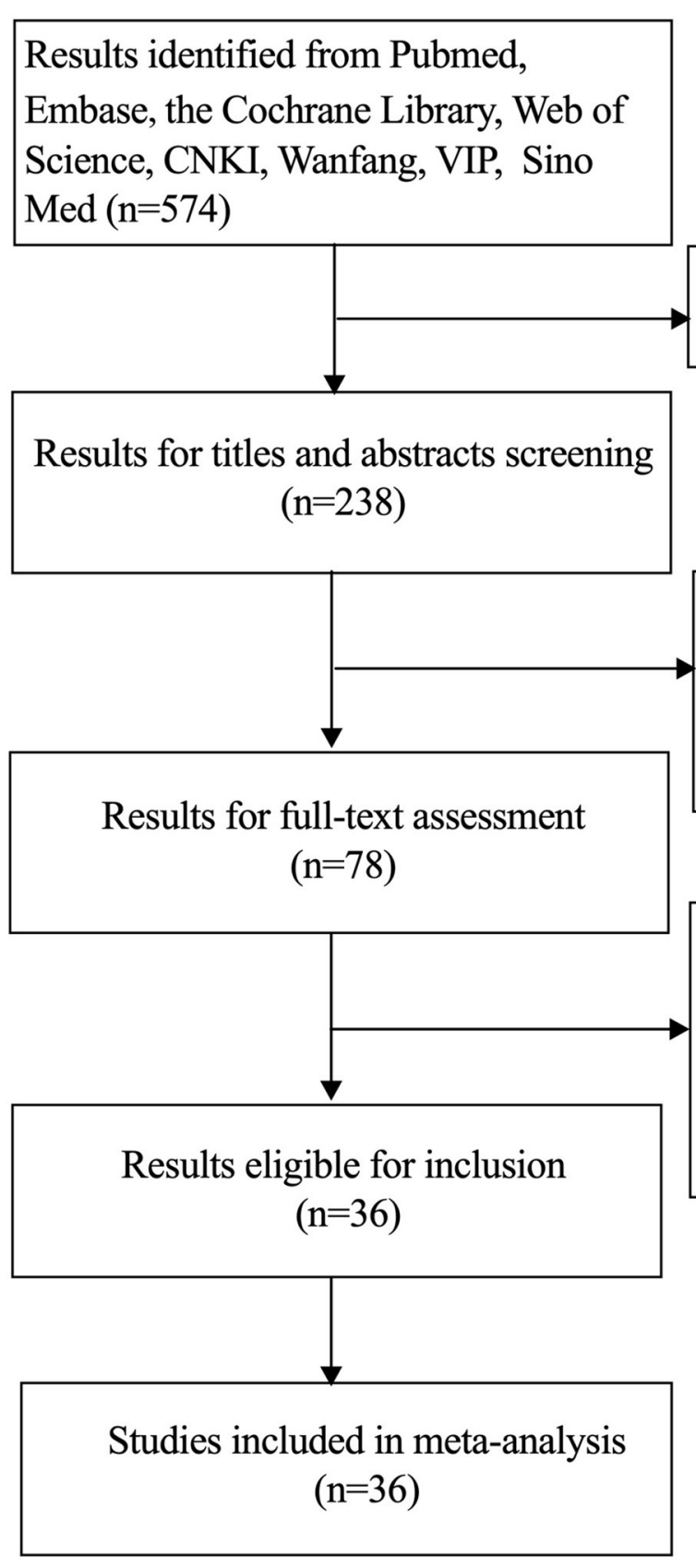

Duplication removal $(\mathrm{n}=336)$

160 records excluded for: -review artical $(\mathrm{n}=28)$ -case report $(n=35)$ -not about traumatic macular hole $(\mathrm{n}=97)$

42 records excluded for:

-lack of adequate information $(n=3)$

-patients had TMH with retinal detachment $(\mathrm{n}=3)$ -outcomes not included $(\mathrm{n}=36)$

no significant heterogeneity $\left(I^{2}<50 \%\right.$ or $\left.P>0.05\right)$, we applied a fixed-effect model to estimate the pooled effect size; otherwise, a random-effect model was employed. Funnel plots were used to detect potential publication bias. A sensitivity analysis was performed to test the robustness of the analysis. A subgroup analysis was conducted to explore the potential heterogeneity among patients in the surgery according to the different surgical procedures. All data synthesis and analysis were performed using Stata version 15.1 .

\section{RESULTS}

\section{Literature Search and Study Selection}

A total of 574 records were identified with the initial search strategy. After removing 336 duplicates, 238 studies were assessed by title and abstract. Thirty-six studies, including 33 retrospective studies and three prospective studies, were selected for our metaanalysis according to the inclusion and exclusion criteria. The details of the search strategy are shown in Figure 1. 
TABLE 1 | Study design and baseline patient characteristics of the surgery group studies.

\begin{tabular}{|c|c|c|c|c|c|c|c|c|c|c|c|c|}
\hline References & Design & Country & $\begin{array}{l}\text { Follow-up } \\
\text { (months) }\end{array}$ & No. of eyes & $\begin{array}{l}\text { Mean age } \\
\text { (years) }\end{array}$ & $\begin{array}{l}\text { Gender } \\
\text { (Male/ } \\
\text { Female) }\end{array}$ & $\begin{array}{l}\mathrm{BCVA} \pm \mathrm{SD} \\
\text { logMAR } \\
\text { (pre/post) }\end{array}$ & $\begin{array}{l}\text { Size of Macular } \\
\text { hole }(\mu \mathrm{m})\end{array}$ & $\begin{array}{l}\text { Interval } \\
\text { from injury } \\
\text { to surgery }\end{array}$ & Operation & $\begin{array}{l}\text { Closure } \\
\text { Rate }\end{array}$ & $\begin{array}{l}\text { VA } \\
\text { improvement } \\
\text { Rate }\end{array}$ \\
\hline Kunikata et al. (3) & Retrospective & Japan & 12.9 & 18 & 18.3 & $15 / 3$ & $\begin{array}{l}0.65 \pm 0.08 / 0.21 \\
\pm 0.07\end{array}$ & $312.5 \pm 170.8$ & $\begin{array}{l}71.7 \pm 44.2 \\
\text { days }\end{array}$ & $\begin{array}{l}\text { PPV + ILM peeling or } \\
\text { ILM flap + SF6 }\end{array}$ & 0.9444 & 1.0000 \\
\hline Chang et al. (13) & Prospective & China & $3.56 \pm 1.32$ & 32 & \multicolumn{2}{|c|}{$31.02 \pm 5.9822 / 10$} & $\begin{array}{l}0.59 \pm 0.12 / 0.14 \\
\pm 0.06\end{array}$ & $650.28 \pm 34.19$ & $1-4$ months & PPV + ILM Peeling & 0.9063 & 0.9375 \\
\hline Ghoraba et al. (14) & ) Retrospective & Egypt & $37 \pm 45$ & 28 & $21.4 \pm 13$ & $23 / 5$ & NA & $757 \pm 221$ & $\begin{array}{l}9 \pm 23.5 \\
\text { months }\end{array}$ & PPV + ILM peeling & 0.7500 & NA \\
\hline Chen et al. (7) & Prospective & China & 6 & 25 & $31.0 \pm 12.5$ & $22 / 3$ & $\begin{array}{l}1.00 \pm 0.35 / 0.56 \\
\pm 0.36\end{array}$ & $512.4 \pm 315.1$ & $\begin{array}{l}20.8 \pm 8.8 \\
\text { days }\end{array}$ & $\begin{array}{l}\text { PPV + ILM peeling + } \\
\text { C3F8/air filling }\end{array}$ & 1.0000 & 0.8800 \\
\hline Fan et al. (15) & Retrospective & China & 3 & 33 & $37.02 \pm 1.35$ & $17 / 16$ & NA & NA & NA & PPV & 0.7879 & 0.4242 \\
\hline Tang et al. (16) & Retrospective & \multicolumn{2}{|c|}{$\begin{array}{l}\text { Australian and } 12 \\
\text { New Zealand }\end{array}$} & 23 & 43.2 & NA & NA & 374 & 117 days & $\begin{array}{l}\text { PPV + ILM peeling (21 } \\
\text { cases) + C3F8/SF6 }\end{array}$ & 0.9130 & 0.4783 \\
\hline Li et al. (17) & Retrospective & China & 12 & 25 & $28.5 \pm 10.1$ & NA & NA & $281.3 \pm 111.3$ & NA & PPV + ILM peeling+air & 0.8000 & 0.2800 \\
\hline Li et al. (17) & Retrospective & China & 12 & 28 & $26.1 \pm 12.9$ & NA & NA & $397.6 \pm 98.2$ & NA & $\begin{array}{l}\text { PPV + ILM peeling + } \\
\text { C3F8 }\end{array}$ & 0.8214 & 0.3214 \\
\hline Fu et al. (18) & Retrospective & China & $4.6 \pm 0.5$ & 30 & $36.4 \pm 3.7$ & NA & $0.12 \pm 0.06 / \mathrm{NA}$ & $648.5 \pm 105.3$ & $\begin{array}{l}8.5 \pm 5.7 \\
\text { days }\end{array}$ & $\begin{array}{l}\text { PPV + ILM peeling + } \\
\text { C3F8 }\end{array}$ & 0.8667 & 0.5000 \\
\hline Li et al. (19) & Retrospective & China & 10 & 16 & $12-45$ & $16 / 0$ & $\begin{array}{l}0.07 \pm 0.01 / 0.33 \\
\pm 0.02\end{array}$ & $477 \pm 183$ & NA & $\begin{array}{l}\text { PPV + ILM peeling + } \\
\text { air }\end{array}$ & 0.8125 & 0.9375 \\
\hline Browne et al. (20) & Retrospective & Egypt & 6 & 16 & $29.95 \pm 9.98$ & $14 / 2$ & $\begin{array}{l}1.1 \pm 0.2 / 0.2 \pm \\
0.13\end{array}$ & $401.44 \pm 34.8$ & NA & $\begin{array}{l}\text { PPV + ILM peeling + } \\
\text { C3F8 }\end{array}$ & 0.9375 & NA \\
\hline Brennan et al. (21) & Retrospective & Switzerland & 12 & 13 & $\begin{array}{l}14.15 \pm \\
2.882\end{array}$ & $10 / 3$ & $\begin{array}{l}0.91 \pm 0.43 / 0.50 \\
\pm 0.17\end{array}$ & NA & $\begin{array}{l}5.38 \pm 3.5 \\
\text { months }\end{array}$ & $\begin{array}{l}\text { PPV + ILM peeling + } \\
\text { C3F8 }\end{array}$ & 0.9231 & 0.9231 \\
\hline Li et al. (22) & Retrospective & China & 12 & 34 & $34.1 \pm 7.4$ & NA & $0.12 \pm 0.07 / \mathrm{NA}$ & $653.6 \pm 123.9$ & $\begin{array}{l}40.8 \pm 20.6 \\
\text { days }\end{array}$ & $\begin{array}{l}\text { PPV + ILM peeling + } \\
\text { C3F8 }\end{array}$ & 0.7059 & 0.3824 \\
\hline Zhu et al. (23) & Retrospective & China & 6 & 28 & $29.01 \pm 7.33$ & $22 / 6$ & $\begin{array}{l}0.086 \pm \\
0.101 / 0.202 \pm \\
0.171\end{array}$ & NA & NA & $\begin{array}{l}\text { PPV + ILM peeling + } \\
\text { C2F6 }\end{array}$ & 0.8571 & 0.6786 \\
\hline $\begin{array}{l}\text { Abou Shousha } \\
\text { et al. (24) }\end{array}$ & Prospective & Egypt & 9 & 12 & $23.25 \pm 14.11$ & $8 / 4$ & NA & $696 \pm 445$ & $\begin{array}{l}3.75 \pm 1.06 \\
\text { months }\end{array}$ & PPV + ILM flap + SF6 & 1.0000 & 0.9167 \\
\hline Chen et al. (25) & Retrospective & China & $4-12$ & 11 & $26.36 \pm 8.43$ & $8 / 3$ & NA & NA & 4-14 months & $\begin{array}{l}\text { PPV + ILM peeling + } \\
\text { air }\end{array}$ & 0.6364 & 0.4545 \\
\hline Yuan et al. (26) & Retrospective & China & 12 & 26 & $32.4 \pm 9.7$ & NA & $\begin{array}{l}0.13 \pm 0.07 / 0.15 \\
\pm 0.07\end{array}$ & $643.3 \pm 125$ & NA & $\begin{array}{l}\text { PPV + ILM peeling + } \\
\text { C3F8 }\end{array}$ & 0.6923 & 0.2692 \\
\hline Tian et al. (27) & Retrospective & China & 12 & 10 & 44.6 & $6 / 4$ & NA & 607.13 & NA & $\begin{array}{l}\text { PPV + ILM peeling + } \\
\text { intraocular tamponade }\end{array}$ & 0.8000 & 0.5000 \\
\hline Hou et al. (28) & Retrospective & China & $1-27$ & 54 & $27.2 \pm 12.4$ & $48 / 6$ & $\begin{array}{l}1.06 \pm 0.39 / 0.84 \\
\pm 0.43\end{array}$ & $598 \pm 227$ & $\begin{array}{l}1-156 \\
\text { months }\end{array}$ & $\begin{array}{l}\text { PPV + ILM peeling + } \\
\text { C3F8/SF6/C2F6 + } \\
\text { platelet concentrate }\end{array}$ & 0.8889 & 0.5185 \\
\hline Wan et al. (29) & Retrospective & China & $6-14$ & 24 & NA & $22 / 2$ & NA & $623 \pm 303$ & 4-24 months & $\begin{array}{l}\text { PPV + ILM peeling + } \\
\text { C3F8 }\end{array}$ & 0.9167 & 0.7083 \\
\hline
\end{tabular}


TABLE 1 | Continued

\begin{tabular}{|c|c|c|c|c|c|c|c|c|c|c|c|c|}
\hline References & Design & Country & $\begin{array}{l}\text { Follow-up } \\
\text { (months) }\end{array}$ & No. of eyes & $\begin{array}{l}\text { Mean age } \\
\text { (years) }\end{array}$ & $\begin{array}{l}\text { Gender } \\
\text { (Male/ } \\
\text { Female) }\end{array}$ & $\begin{array}{l}\mathrm{BCVA} \pm \mathrm{SD} \\
\text { logMAR } \\
\text { (pre/post) }\end{array}$ & $\begin{array}{l}\text { Size of Macular } \\
\text { hole }(\mu \mathrm{m})\end{array}$ & $\begin{array}{l}\text { Interval } \\
\text { from injury } \\
\text { to surgery }\end{array}$ & Operation & $\begin{array}{l}\text { Closure } \\
\text { Rate }\end{array}$ & $\begin{array}{l}\text { VA } \\
\text { improvement } \\
\text { Rate }\end{array}$ \\
\hline Ghoraba et al. (30) & Retrospective & Egypt & $14.46 \pm 3.43$ & 13 & $26.54 \pm 5.68$ & $9 / 4$ & $0.061 / 0.433$ & NA & NA & $\begin{array}{l}\text { PPV+ILM } \\
\text { peeling+C3F8 }\end{array}$ & 0.9231 & NA \\
\hline Qu et al. (31) & Retrospective & China & $\begin{array}{l}96 \pm 131 \\
\text { days }\end{array}$ & 95 & $26.6 \pm 13.5$ & $87 / 8$ & $\begin{array}{l}1.1 \pm 0.45 / 0.83 \pm \\
0.40\end{array}$ & $644.2 \pm 270.5$ & $\begin{array}{l}9.8 \pm 21.8 \\
\text { months }\end{array}$ & $\begin{array}{l}\text { PPV + ILM peeling (90 } \\
\text { cases) or not + } \\
\text { C3F8/SF6/C2F6 + } \\
\text { platelet concentrate (85 } \\
\text { cases) }\end{array}$ & $\begin{array}{l}1.0000 \\
5\end{array}$ & 0.7263 \\
\hline Ovali et al. (32) & Retrospective & Turkey & NA & 14 & $40.4 \pm 14.4$ & NA & NA & 425 & NA & $\begin{array}{l}\text { PPV + ILM peeling + } \\
\text { C3F8 (13 } \\
\text { cases)/silicone-oil (1 } \\
\text { case) }\end{array}$ & 0.9286 & 0.8571 \\
\hline Chen et al. (33) & Retrospective & China & 8 & 11 & NA & NA & & & & PPV + ILM peeling+air & 0.6364 & 0.7273 \\
\hline Gong (34) & Retrospective & China & $3-12$ & 14 & $\begin{array}{l}37.93 \pm \\
12.92\end{array}$ & $12 / 12$ & $\begin{array}{l}0.058 \pm \\
0.044 / 0.22 \pm 0.21\end{array}$ & $628.79 \pm 183.33$ & $\begin{array}{l}45.36 \pm \\
45.24 \text { days }\end{array}$ & PPV + ILM peeling & 1.000 & 0.7143 \\
\hline Wu et al. (35) & Retrospective & America & $12.5 \pm 16.4$ & 13 & 10 & $10 / 3$ & NA & NA & $\begin{array}{l}2.9 \pm 2.0 \\
\text { months }\end{array}$ & $\begin{array}{l}\text { Plasmin } \\
\text { Enzyme-Assisted PPV } \\
+ \text { ILM peeling ( } 3 \text { cases) } \\
+ \text { C3F8/ silicone-oil }\end{array}$ & 0.9231 & 0.9167 \\
\hline Ma et al. (36) & Retrospective & China & $3-6$ & 8 & 24.13 & $7 / 1$ & NA & NA & NA & $\begin{array}{l}\text { PPV + ILM peeling + } \\
\text { C3F8 }\end{array}$ & 0.8750 & 0.7500 \\
\hline Liu and Gong (37) & Retrospective & China & $4-24$ & 12 & $18-65$ & $9 / 3$ & NA & NA & 3-12 months & $\begin{array}{l}\text { PPV + ILM peeling (3 } \\
\text { cases) + C3F8/SF6 }\end{array}$ & 0.8333 & 0.7500 \\
\hline Kuhn et al. (38) & Retrospective & Hungary & 14 & 17 & 26 & $15 / 2$ & NA & NA & 2.5 months & $\begin{array}{l}\text { PPV + ILM peeling + } \\
\text { SF6 }\end{array}$ & 1.0000 & 0.9412 \\
\hline Johnson et al. (39) & Retrospective & America & 11 & 25 & 23 & $20 / 5$ & NA & NA & NA & $\begin{array}{l}\text { PPV + ILM peeling + } \\
\text { C3F8 }\end{array}$ & 0.9600 & 0.8400 \\
\hline $\begin{array}{l}\text { García-Arumí et al. } \\
\text { (40) }\end{array}$ & Retrospective & Spain & 13 & 14 & 19 & $11 / 3$ & NA & NA & $1-6$ weeks & $\begin{array}{l}\mathrm{PPV}+\text { platelet } \\
\text { concentrate + SF6 }\end{array}$ & 0.9286 & 0.9286 \\
\hline Rubin et al. (41) & Retrospective & America & 12.1 & 12 & 15 & $11 / 1$ & NA & NA & 19 weeks & PPV + TGF $-\beta+$ C3F8 & 0.9167 & 0.6667 \\
\hline
\end{tabular}

BCVA, best-corrected visual acuity; C2F6, hexafluoroethane; C3F8, perfluoropropane; SF6, sulfur hexafluoride; ILM, internal limiting membrane; logMAR, logarithm of the minimal angle of resolution; NA, not available; PPV, pars plana vitrectomy; VA, visual acuity. 
TABLE 2 | Study design and baseline patient characteristics of observation group studies.

\begin{tabular}{|c|c|c|c|c|c|c|c|c|c|c|c|}
\hline References & Design & Country & $\begin{array}{c}\text { Follow-up } \\
\text { (months) }\end{array}$ & $\begin{array}{c}\text { No. of } \\
\text { Patients }\end{array}$ & $\begin{array}{c}\text { Mean age } \\
\text { (years) }\end{array}$ & $\begin{array}{c}\text { Gender } \\
\text { (male/female) }\end{array}$ & $\begin{array}{c}\text { BCVA } \pm \text { SD } \\
\text { logMAR } \\
\text { (pre/post) }\end{array}$ & $\begin{array}{l}\text { Size of macular } \\
\text { hole }(\mu \mathrm{m})\end{array}$ & $\begin{array}{l}\text { Time of hole } \\
\text { closure }\end{array}$ & Closure Rate & $\begin{array}{c}\text { VA improvement } \\
\text { Rate }\end{array}$ \\
\hline Chen et al. (7) & Prospective & China & 6 & 15 & $33.1 \pm 11.6$ & $14 / 1$ & $\begin{array}{c}1.11 \pm 0.48 / 0.75 \\
\pm 0.4\end{array}$ & $423.2 \pm 242.9$ & $2.5 \pm 1.6$ months & 0.6667 & 0.4667 \\
\hline Fan et al. (15) & Retrospective & China & 3 & 30 & $37.28 \pm 1.40$ & $16 / 14$ & NA & NA & NA & 0.5667 & 0.4333 \\
\hline Fu et al. (18) & Retrospective & China & $4.6 \pm 0.5$ & 26 & $35.9 \pm 3.4$ & NA & $0.13 \pm 0.08 / \mathrm{NA}$ & $653.8 \pm 94.7$ & 12 months & 0.4231 & 0.4615 \\
\hline Li et al. (22) & Retrospective & China & 12 & 27 & $31.2 \pm 5.5$ & $19 / 8$ & $0.13 \pm 0.06 / \mathrm{NA}$ & $632.5 \pm 82.4$ & NA & 0.4074 & 0.4074 \\
\hline Yuan et al. (26) & Retrospective & China & 12 & 21 & $26.1 \pm 10.0$ & NA & NA & $490 \pm 86.9$ & $51.0 \pm 12.6$ days & 0.3333 & 0.3333 \\
\hline Chen et al. (42) & Retrospective & China & 6 & 27 & $26.2 \pm 10.7$ & $23 / 4$ & $\begin{aligned} 1.36 & \pm 0.74 / 1.01 \\
& \pm 0.60\end{aligned}$ & NA & NA & 0.3704 & 0.3333 \\
\hline Tian et al. (27) & Retrospective & China & 12 & 12 & 35 & $8 / 4$ & NA & NA & NA & 0.6667 & 0.5000 \\
\hline Hou et al. (43) & Retrospective & China & 16 & 30 & 32 & $27 / 3$ & NA & NA & NA & 0.1000 & 0.4000 \\
\hline Chen et al. (33) & Retrospective & China & 12 & 30 & NA & NA & NA & NA & 2.83 months & 0.3000 & 0.3667 \\
\hline Li et al. (44) & Retrospective & China & 14 & 28 & 30.1 & $25 / 3$ & NA & NA & 4-5 months & 0.1071 & 0.2857 \\
\hline Jin et al. (45) & Retrospective & China & $\begin{array}{c}20.72 \pm \\
11.61\end{array}$ & 11 & $19.55 \pm 8.18$ & $10 / 1$ & NA & NA & NA & 0.2727 & 0.2727 \\
\hline $\begin{array}{l}\text { Yamashita et al. } \\
\text { (46) }\end{array}$ & Retrospective & Japan & 8.4 & 18 & 14.6 & NA & NA & NA & $\begin{array}{c}1 \text { week to } 4 \\
\text { months }\end{array}$ & 0.4444 & 0.4444 \\
\hline
\end{tabular}




\section{Characteristics of the Study Samples}

Thirty-two studies, as shown in Table 1, reported data on patients ( $n=734$ ) who underwent vitrectomy. For the surgery group patients, vitrectomies were performed using adjunctive therapies, including ILM peeling or flap, platelet concentrate or TGF- $\beta$, and gas or silicon oil tamponade. Most of the patients were males, with a similar proportion in both groups. The mean age of the surgery group patients was 26.95 years $(n=671$; range, 1-69 years). The mean follow-up time was $10.41 \pm 6.48$ months ( $n=662$; median, 12 ; range, $3-45$ months). The interval from injury to surgery ranged from 1 week to 120 months, and the average size of the TMH was $628.84 \mu \mathrm{m}(n=358$; range, 64$1,588 \mu \mathrm{m})$. The mean preoperative and postoperative BCVA were $0.87 \log$ MAR $(n=247)$ and $0.48 \log$ MAR $(n=247)$, respectively. The pooled event rate for BCVA improvement in the surgery group was 0.39 (95\% CI, 0.33-0.46).

For the observation group, 12 studies and 275 patients were analyzed (Table 2). The average age of the observation group patients was 30.36 years ( $n=157$; range, 9-49 years), the mean follow-up time was $10.56 \pm 5.15$ months $(n=275$, median, 12; range, 3-48 months), and the average size of the TMH was $561.10 \mu \mathrm{m}(n=93$; range, 553.6-681.4 $\mu \mathrm{m})$. The percentage of patients who achieved TMH closure in $<6$ months was $>80 \%$.

\section{Pooled Rates of Closure and VA Improvement for the Surgery Group}

In the surgery group, the pooled rates of TMH closure and VA improvement were reported in 31 studies (709 eyes) and 28 studies (651 eyes), respectively. The random-model pooled rate for TMH closure was 0.9 (95\% CI, 0.85-0.94, Figure 2A), while that for VA improvement was 0.72 (95\% CI, 0.63-0.80). There was high heterogeneity between the studies $\left(I^{2}=64.19 \%, P<\right.$ $0.05 ; I^{2}=81.13 \%, P<0.05$, Figure 2B). The Funnel plots did not reveal evidence of publication bias (Supplementary Material). For the TMH closure rate, sensitivity analysis suggested that one study (31) may have been a potential source of heterogeneity (Supplementary Material). After excluding this study, the pooled rate of TMH closure in the remaining 30 studies was 0.89 (95\% CI, 0.85-0.92, $I^{2}=40.10 \%, P<0.05$ ).

The results of the subgroup analyses are shown in Table 3. There was no significant difference in TMH closure rate between subgroups stratified by different types of operation. Statistically significant effects of the subgroups were identified for VA improvement rate and BCVA logMAR improvement $(P<0.05$ for heterogeneity between groups).

The pooled event rate of TMH closure was higher in the pars plana vitrectomy $(\mathrm{PPV})+$ ILM peeling + perfluoropropane (C3F8)/sulfur hexafluoride (SF6)/hexafluoroethane (C2F6) group (0.88, 95\% CI: $0.82-0.93, I^{2}=37.22 \%$, Table 3$)$ than in the PPV + ILM peeling + air group (0.75, 95\% CI: $0.63-$ $0.86, I^{2}=0 \%$, Table 3). However, there was still unexplained heterogeneity $\left(I^{2}>50 \%\right)$ between subgroups in the VA improvement rate, which the subgroup analysis could not completely explain. For BCVA logMAR improvement, the patients in the PPV+ILM peeling + platelet concentrate + intraocular tamponade group had a better BCVA improvement
(0.25, 95\% CI: $0.16-0.35, I^{2}=0 \%$, Table 3$)$ than those in the PPV + ILM peeling + intraocular tamponade group $(0.45$, 95\% CI: $0.41-0.48, I^{2}=0 \%$, Table 3). Meta-regression revealed that different types of operations affected the results of BCVA $\log$ MAR improvement.

\section{Pooled Rates of Closure and VA Improvement for the Observation Group}

For the observation group, the rate of $\mathrm{TMH}$ closure and VA improvement was reported in 12 studies (275 eyes). The random-model pooled rate for $\mathrm{TMH}$ closure was $0.37(95 \%$ CI, 0.26-0.48). There was high heterogeneity among the studies $\left(I^{2}=71.07 \%, P<0.05\right)$ (Figure 3A). The included studies were not randomized controlled trials (RCTs), which may be a potential source of heterogeneity. Publication bias was not assessed. Sensitivity analysis showed that the results were robust (Supplementary Material). The pooled event rate for VA improvement was 0.39 (95\% CI, 0.33-0.45; fixed model) with no heterogeneity between the studies $\left(I^{2}=0.00 \%, P>0.05\right)$ (Figure 3B).

\section{Adverse Effects}

Mild vitreous hemorrhage was noted in one patient one day after vitrectomy surgery, which resolved within 1 week (40). Vitreoretinal surgery combined with the use of intraocular gases can result in elevated postoperative intraocular pressure (IOP) and cataract formation. Three studies reported increased IOP after surgery, but it was controlled within the normal range after medication $(30,37)$. In six studies, 24 patients developed cataract formation or acceleration during the follow-up period $(17,23,29,30,37)$. After the operation, three patients from two studies developed retinal detachment $(17,31)$. One of the reasons for this was an improper surgical operation. None of the patients in these studies developed endophthalmitis. In the observation group, 17 eyes developed obvious hole enlargement and two eyes had retinal detachment $(43,44)$.

\section{DISCUSSION}

\section{Summary of the Main Results}

We obtained several results by combining the existing evidence. First, although the studies included in our meta-analysis were of moderate or high methodological quality, there were no RCTs of TMH treatment, which may have led to a lack of convincing results. Second, the rates of TMH closure and VA improvement were significantly higher in the surgery group than in the observation group. This evidence may represent the best available support for treating patients with vitrectomy. Third, $\mathrm{TMH}$ patients were younger and mainly males, and over $80 \%$ of them showed closure with observation in $<6$ months. Raised IOP and cataracts are common postoperative complications, but these will not be severely adverse if immediate and proper treatment is adopted. 


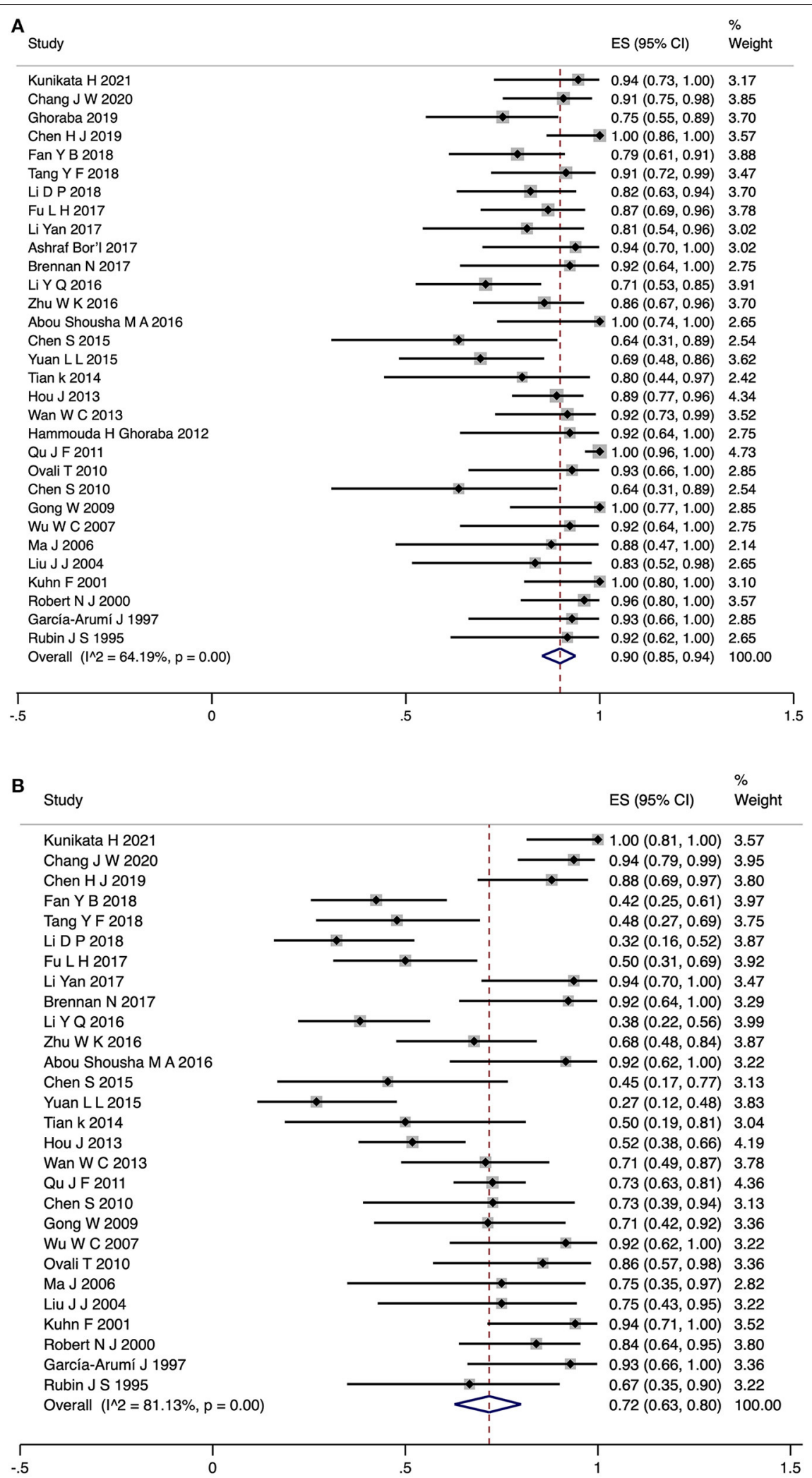

FIGURE 2 | Forest plot for TMH closure rate (A) and VA improvement rate (B) of surgery group patients. TMH, traumatic macular hole; VA, visual acuity. 
TABLE 3 | Subgroup analysis for outcomes.

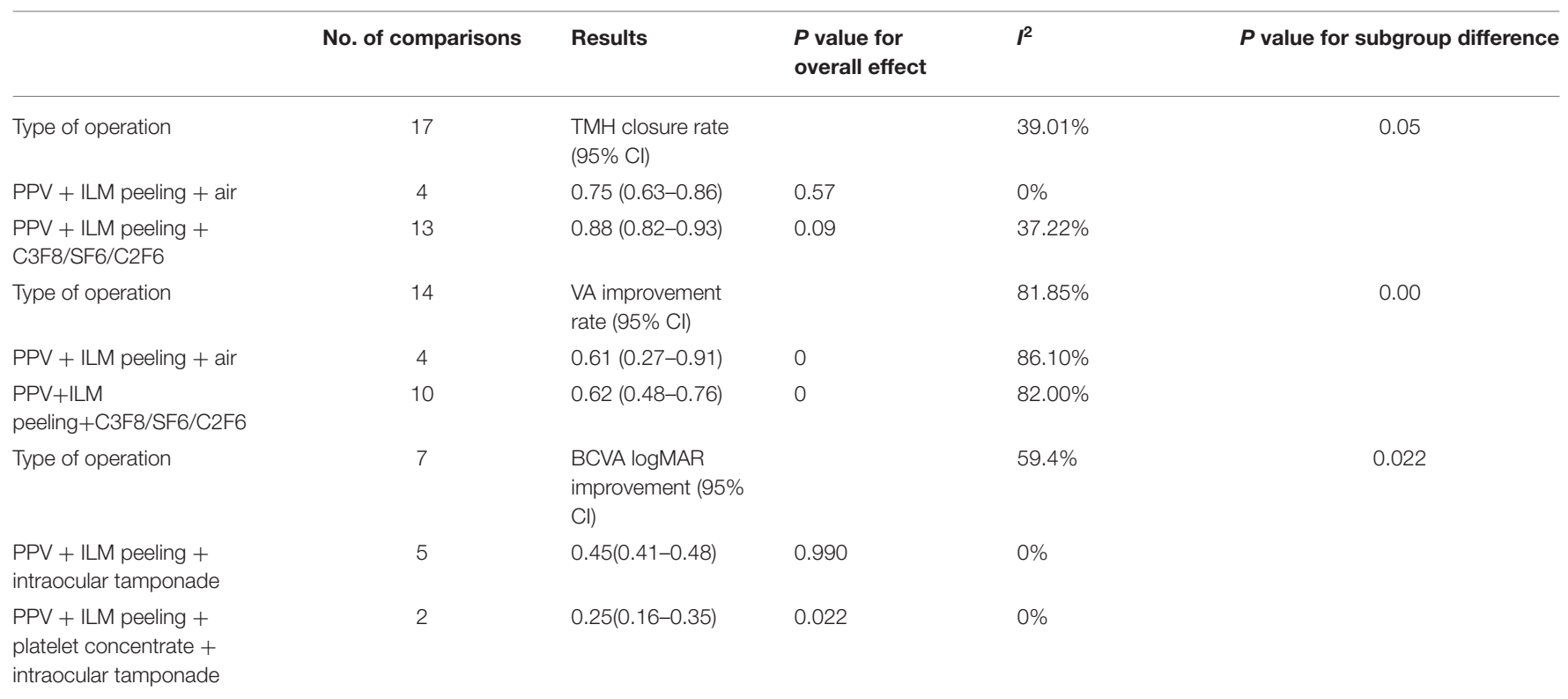

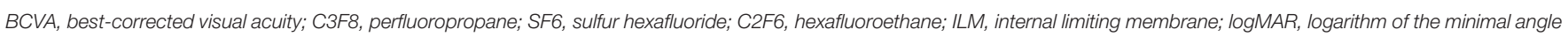
of resolution; PPV, pars plana vitrectomy; $T M H$, traumatic macular hole; $V A$, visual acuity.

\section{Rate of Closure and VA Improvement According to Vitrectomy Surgery}

In this systematic review, the TMH closure rate ranges from 0.63 to 1.0 with a pooled event rate of 0.90 , while the VA improvement rate ranges from 0.28 to 1.0 , with a pooled event rate of 0.72 in patients undergoing surgery. According to the study by Wang and Peng (47), the closure and VA improvement rates were 0.83 and 0.84 , respectively, so that our results are similar to theirs, and showed that vitrectomy surgery seems to be a more effective method than observation for TMH treatment. The widespread use of optical coherence tomography (OCT) can offer further insight into the nature of TMH and shed light on the possible reasons for this Miller et al. (2) reported that an intact ellipsoid zone in closed holes tended to correlate with improved final visual acuity. A multicenter prospective comparative study showed that there were no significant differences in the length of the photoreceptor IS/OS junction (ellipsoid zone) defect and the final BCVA between the surgically closed cases and spontaneously closed cases, with $80 \%$ of the patients showing spontaneous hole closure within 3 months (7). Thus a 3month observation period after injury may be an alternative modality for TMH management. Therefore, many researchers suggested that vitreous surgery should be carried out in 3 months to prevent severe photoreceptor damages. The closure rate was higher than the VA improvement rate in the surgery group, and the differences between anatomical and functional outcomes may be associated with different preoperative retinal pathologies and ocular complications (1). The study by $\mathrm{Qu}$ et al. (31), which reported a TMH closure rate of 1.0 , may be a potential source of heterogeneity. The reason for the high closure rate may be associated with the use of adjunctive therapy (platelet concentrate).

The underlying mechanism of TMH formation is unclear. One type forms immediately after ocular trauma, with the foveal rupture causing acute vision loss. Another type may result from the development of macular edema and cysts, which may induce delayed-onset TMH formation. With the regression of macular edema, shrinkage and closure of the hole may occur. Glial cell proliferation and epiretinal membrane formation are often the causes of a persistent open hole. Therefore, vitrectomy with membrane peeling might be helpful and is a standard surgical procedure for treating TMH (6). Currently, PPV, ILM peeling or flap, and intraocular gas or silicone oil tamponade are the most commonly employed surgical procedures for TMH treatment (5). Ghoraba et al. concluded that gas tamponade is more successful than silicone oil tamponade for the anatomical closure and VA improvement of TMH (30). Intraocular gas tamponade is a crucial component of the surgical procedure for TMH repair. Higher rates of TMH closure were observed with C3F8, SF6, or C2F6 ocular tamponades, which could result from the extended amount of time the C3F8, SF6, or C2F6 lasts in the vitreous cavity. In this meta-analysis, the TMH closure rate and VA improvement rate in the C3F8/SF6/C2F6 tamponade group showed better outcomes than that in the air tamponade group.

Adjunctive therapies are often used together with surgery to accelerate hole closure $(28,40,41)$. Rubin et al. used TGF- $\beta 2$ in 12 eyes during vitrectomy and finally achieved a closure rate of $67 \%$ in eight eyes (41). Garca-Arum et al. found that the intraoperative application of platelet concentrate in combination 


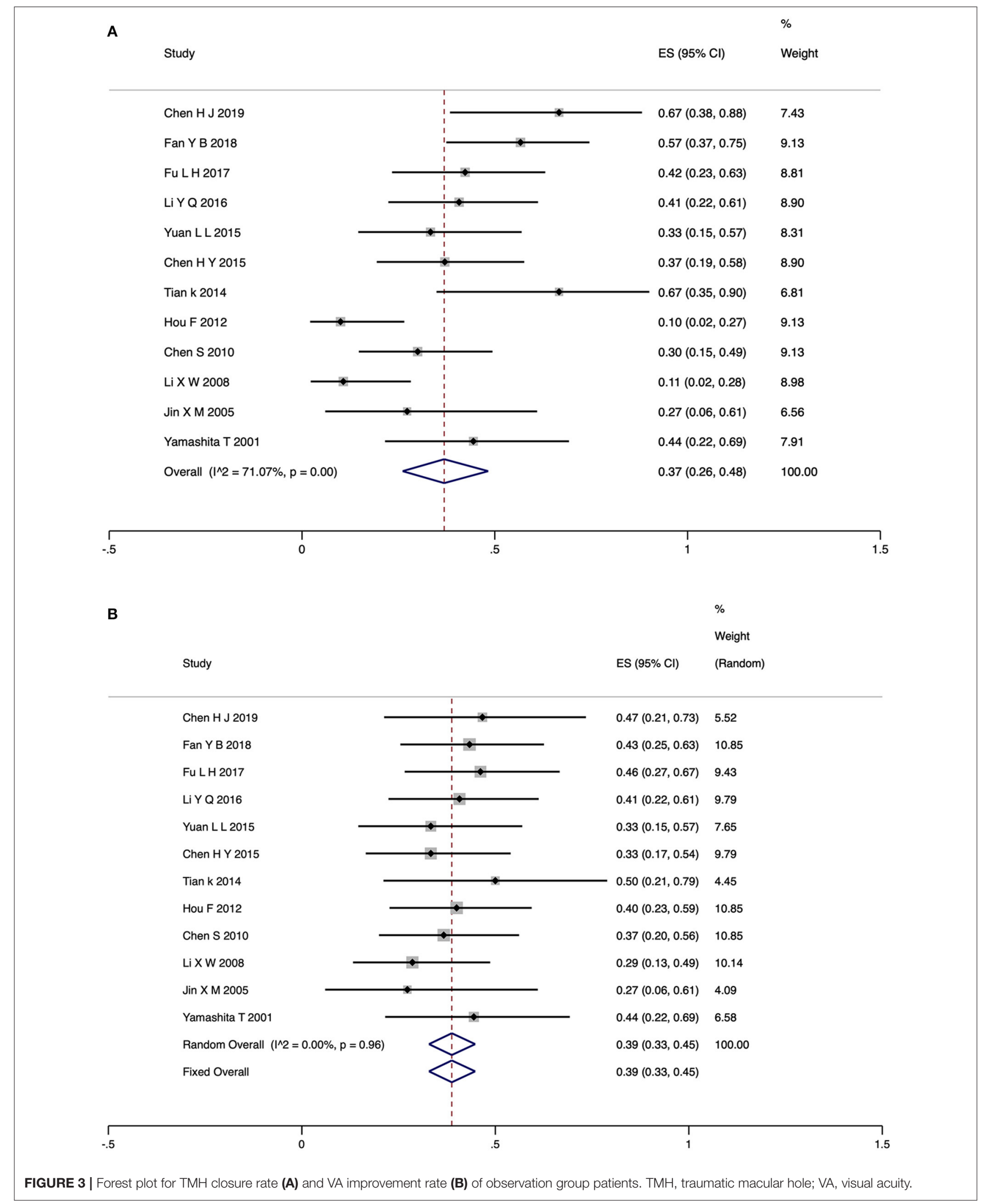


with vitrectomy may help improve anatomic and visual outcomes (40). As shown by our meta-analysis, platelet concentrate was a potential factor that affected visual improvement. However, studies are unlikely to be designed to evaluate adjunctive therapies, which are also seldom implemented today.

\section{Spontaneous TMH Closure}

$\mathrm{TMH}$ has been shown to close without any treatment, usually between 1 and 6 months after the trauma incident $(8,9)$. The closure and VA improvement rates were 0.37 and 0.39 , respectively, similar to those reported in previous publications. In our meta-analysis, over $80 \%$ of the patients with TMH achieved closure within 6 months. The mechanism of spontaneous closure is of great interest. Why does TMH have a higher spontaneous closure rate than other types of $\mathrm{MH}$ ? The fact that $\mathrm{TMH}$ patients are young and have a healthy vitreous gel and a firm vitreofoveal attachment may account for the high rate of spontaneous closure. Indeed, young age, small hole size, cystic edema at the edge of the $\mathrm{MH}$, and no posterior vitreous detachment have been recognized as possible features affecting spontaneous closure $(7,48)$.

In addition, it should be noted that 17 eyes developed obvious hole enlargement and two eyes showed retinal detachment (43, 44). In five studies, 134 patients received supportive drugs (such as Sanqi Panax notoginseng for injection, compound anisodine hydrobromide injection, inosine tablets, or iodizedlecithin) to prepare the optic nerve and promote retinal microcirculation. However, experimental or clinical proof about their efficacy is lacking.

\section{Limitations}

This meta-analysis has some limitations. First, high heterogeneity existed in some outcomes, and many factors could have led to heterogeneity, such as the size of $\mathrm{MH}$, different types of surgery, and interval from injury to surgery. However, the complete data were hardly accessed for subgroup analysis, and the factors were relative to treatment decisions. Second, since the studies included were retrospective and prospective observational studies and not RCTs, the comparison between the surgery and observation

\section{REFERENCES}

1. Liu W, Grzybowski A. Current management of traumatic macular holes. J Ophthalmol. (2017) 4:1-8. doi: 10.1155/2017/1748135

2. Miller J B, Yonekawa Y, Eliott D, Kim IK, Kim LA, Loewenstein JI, et al. Longterm follow-up and outcomes in traumatic macular holes. Am J Ophthalmol. (2015) 160:1225-58. doi: 10.1016/j.ajo.2015.09.004

3. Kunikata H, Osada U, Abe T, Nakazawa T. Efficacy of early microincision vitrectomy surgery in traumatic macular hole. Graefes Arch Clin Exp Ophthalmol. (2021) 259:2451-4. doi: 10.1007/s00417-02105139-7

4. Bor'i A, Al-Aswad MA, Saad AA, Hamada D, Mahrous A. Pars plana vitrectomy with internal limiting membrane peeling in traumatic macular hole: $14 \%$ perfluoropropane (C3F8) versus silicone oil tamponade. J Ophthalmol. (2017) 2017:3917696. doi: 10.1155/2017/ 3917696

5. Coca M, Makkouk F, Picciani R, Godley B, Elkeeb A. Chronic traumatic giant macular hole repair with autologous platelets. Cureus. (2017) 9:e955. doi: 10.7759/cureus.955 groups was based on data with a discrepant baseline. Therefore, given the limitations mentioned above, RCTs are needed in the future to evaluate the effectiveness and safety of surgery and observation for TMH. Therefore, we will update our metaanalysis if RCTs are performed in future.

\section{CONCLUSIONS}

In conclusion, our systematic review and meta-analysis provides evidence that, compared with observation, surgery leads to higher TMH closure and VA improvement rates. Vitrectomy is an effective and safe treatment method for TMH. The management guidelines for $\mathrm{TMH}$ in pediatric patients and the factors affecting the related outcomes need further clarification.

\section{DATA AVAILABILITY STATEMENT}

The original contributions presented in the study are included in the article/Supplementary Material, further inquiries can be directed to the corresponding author.

\section{AUTHOR CONTRIBUTIONS}

QZ, YX, and HL: conceptualization and design. QZ, HF, and ZF: literature search, data extraction, quality assessment, and statistical analysis. QZ: manuscript writing. HY: supervision. All authors approved the final version of the manuscript.

\section{FUNDING}

This work was supported by the National Nature Science Foundation of China (Grant Number: 81774371).

\section{SUPPLEMENTARY MATERIAL}

The Supplementary Material for this article can be found online at: https://www.frontiersin.org/articles/10.3389/fmed. 2021.735968/full\#supplementary-material
6. Miller JB, Yonekawa Y, Eliott D, Vavvas DG. A review of traumatic macular hole: diagnosis and treatment. Int Ophthalmol Clin. (2013) 53:5967. doi: 10.1097/IIO.0b013e3182a26efe

7. Chen HJ, Jin Y, Shen LJ, Wang Y, Li ZY, Fang XY, et al. Traumatic macular hole study: a multicenter comparative study between immediate vitrectomy and six-month observation for spontaneous closure. Ann Transl Med. (2019) 7:726-726. doi: 10.21037/atm.2019.12.20

8. Valmaggia C, Pfenninger L, Haueter I. Spontaneous closure of a traumatic macular hole. Klin Monbl Augenheilkd. (2009) 226:361-2. doi: 10.1055/s-0028-1109251

9. Karaca U, Durukan HA, Mumcuoglu T, Erdurman C, Hurmeric V. An unusual complication of blunt ocular trauma: a horseshoe-shaped macular tear with spontaneous closure. Indian J Ophthalmol. (2014) 62:5013. doi: 10.4103/0301-4738.121138

10. Gao M, Liu K, Lin Q, Liu H. Management modalities for traumatic macular hole: a systematic review and single-arm meta-analysis. Curr Eye Res. (2017) 42:287-96. doi: 10.1080/02713683.2016.1175021

11. Stroup DF. Meta-analysis of observational studies in epidemiology:a proposal for reporting. JAMA. (2000) 283:2008-8. doi: 10.1001/jama.283.15.2008 
12. Zeng X, Zhang Y, Kwong JS, Zhang C, Li S, Sun F, et al. The methodological quality assessment tools for preclinical and clinical studies, systematic review and meta-analysis, and clinical practice guideline: a systematic review. J Evid Based Med. (2015) 8:2-10. doi: 10.1111/jebm.12141

13. Chang JW, Pan JH, Yin JJ. Comparison of the efficacy between two different surgical methods for traumatic macular hole. Chin J Ocul Traum Occupat Eye Dis. (2020) 42:407-12.

14. Ghoraba HH, Leila M, Ghoraba H, Heikal MA, Elgemai EEM. Comparative study between pars plana vitrectomy with internal limiting membrane peel and pars plana vitrectomy with internal limiting membrane flap technique for management of traumatic full thickness macular holes. J Ophthalmol. (2019) 2019:1959082. doi: 10.1155/2019/1959082

15. Fan YB, Xiao Y, Lv WJ, Cai XH, Gu YX. Comparative analysis of surgical and non-surgical treatment on traumatic macular hole. J Hunan Univ Chinese Med. (2018) 38:161.

16. Tang YF, Chang A, Campbell WG, Connell PP, Essex RW. Surgical management of traumatic macular hole: optical coherence tomography features and outcomes. Retina. (2020) 40:290-8. doi: 10.1097/IAE.0000000000002382

17. Li DP, Yuan LL, Zhang DC. Efficacy of different vitreous tamponade for the treatment of traumatic macular holes. Chinese J Ocular Trauma Occup Eye Dis. (2018) 40:1-4. doi: 10.3760/cma.j.issn.2095-1477.2018.01.001

18. Fu LH, Lei F. Comparison on clinical efficacy of surgical treatment and conservative treatment for traumatic macular hole. Chinese J Ocular Trauma Occup Eye Dis. (2017) 39:1668. doi: 10.3760/cma.j.issn.2095-1477.2017.03.002

19. Li Y, Tao M, Li YT. Clinical observation of vitrectomy combined with room air-filled on traumatic macular hole. Chinese J Pract Ophthalmol. (2017) 35: 813-6. doi10.3760/j.issn.1006-4443.2017.08.016

20. Browne AW, Conti FF, Kaiser PK, Singh RP. Surgical repair of traumatic macular holes with extreme macular pathology using an internal limiting membrane flap technique. Ophthalmic Surg Lasers Imaging Retina. (2018) 49:E143-6. doi: 10.3928/23258160-20181002-17

21. Brennan N, Reekie I, Khawaja AP, Georgakarakos N, Ezra E. Vitrectomy, inner limiting membrane peel, and gas tamponade in the management of traumatic paediatric macular holes: a case series of 13 patients. Ophthalmologica. (2017) 238:119-23. doi: 10.1159/000477177

22. Li YQ Ji XT, Xu C, Hu JQ. Comparison of application effect of surgical and non-surgical therapy in patients with traumatic macular hole. Chinese J Pract Ophthalmol. (2016) 34:881-3. doi: 10.3760/cma.j.issn.1006-4443.2016.08.029

23. Zhu WK. Observation of vitrectomy and inner retinal membrane peeling in the treatment of traumatic macular hole. China Pract Med. (2016) 11:667. doi: 10.14163/j.cnki.11-5547/r.2016.09.039

24. Abou Shousha MA. Inverted internal limiting membrane flap for large traumatic macular holes. Medicine. (2016) 95:e2523. doi: 10.1097/MD.0000000000002523

25. Chen S, Zhao TY, Qin B, Zhang T. Clinical efficacy of air tamponade during vitrectomy for the treatment of traumatic macular hole. Chinese J Pract Ophthalmol. (2016) 34:881-3. doi: 10.1155/2020/ 4819468

26. Yuan LL, Han JD Li XR. Clinical analysis of 47 cases with traumatic macular hole resulted from ocular contusion. Chinese J Ocular Fundus Dis. (2015) 31:45-8. doi: 10.3760/cma.j.issn.1005-1015.2015.01.012

27. Tian K. Clinical observation of conservative and surgica treatment of traumatic macular hole. Changchun: Jilin University. (2014).

28. Hou J, Jiang YR. An analysis of the prognosis and factors of vitrectomy for a traumatic macular hole. Chinese J Optom Ophthalmol Vis Sci. (2013) 15:26-9. doi: 10.3760/cma.j.issn.1674-845X.2013.01.007

29. Wan WC, Zhang XD Li XD, Zhang NN, Jin XM. Traumatic macular hole treated by inner limiting membrane peeling with vitreous cutting operation. Recent Adv Ophthalmol. (2013) 33:949-51.

30. Ghoraba HH, Ellakwa AF, Ghali AA. Long term result of silicone oil versus gas tamponade in the treatment of traumatic macular holes. Clin Ophthalmol. (2012) 6:49-53. doi: 10.2147/OPTH.S22061

31. Qu JF Li XX, Jiang YR, Zhao MW, Yin H. A clinical study of vitrectomy for traumatic macular hole. Chinese J Pract Ophthalmol. (2011) 29:113942. doi: 10.3760/cma.j.issn.1006-4443.2011.11.011
32. Ovali T. Ovali, Erkul SZ, ad BY, Akaray K. Surgical treatment of traumatic macular hole. Retina-Vitreus. (2010) 18:40-5.

33. Chen S. The study on the prognosis of anatomy and visual function for traumatic rmacular hole. Changchun: Jinan University. (2010).

34. Gong W. The Clinical Treatment ofTraumatic Macular Hole. Wenzhou: Wenzhou Medical University (2009).

35. Wu WC, Drenser KA, Trese MT, Williams GA, Capone A. Pediatric traumatic macular hole: results of autologous plasmin enzyme-assisted vitrectomy. Am J Ophthalmol. (2007) 144:668-72. doi: 10.1016/j.ajo.2007.07.027

36. Ma J, Wang WZ, Jin XM, Yang JX. A clinical study of vitrectomy and internal limiting membrane peeling for traumatic macular hole. Chinese J Pract Ophthalmol. (2006) 4:416-8. doi: 10.3760/cma.j.issn.1006-4443.2006.04.022

37. Liu JJ, Gong XZ. Vitrectomy for traumatic macular hole. Chinese J Ocular Trauma Occup Eye Dis. (2004) 26:3001. doi: 10.3760/cma.j.issn.2095-1477.2004.05.005

38. Kuhn F, Morris R, Mester V, Witherspoon CD. Internal limiting membrane removal for traumatic macular holes. Ophthalmic Surg Lasers. (2001) 32:30815. doi: 10.3928/1542-8877-20010701-09

39. Johnson RN, McDonald HR, Lewis H, Grand MG, Murray TG, Mieler WF, et al. Traumatic macular hole: observations, pathogenesis, and results of vitrectomy surgery. Ophthalmology. (2001) 108: 853-7. doi: 10.1016/S0161-6420(00)00650-3

40. Garca-Arum J, Corcostegui B, Cavero L, Sararols L. The role of vitreoretinal surgery in the treatment of posttraumatic macular hole. Retina. (1997) 17:3727. doi: 10.1097/00006982-199717050-00003

41. Rubin JS, Glaser BM, Thompson JT, Sjaarda RN, Pappas SS Jr, Murphy RP. Vitrectomy, fluid-gas exchange and transforming growth factor-beta-2 for the treatment of traumatic macular holes. Ophthalmology. (1995) 102:18405. doi: 10.1016/S0161-6420(95)30786-5

42. Chen H, Chen W, Zheng K, Peng K, Xia H, Zhu L. Prediction of spontaneous closure of traumatic macular hole with spectral domain optical coherence tomography. Sci Rep. (2015) 5:12343. doi: 10.1038/srep 12343

43. Hou F, Lu N, Zhang L. Clinical study on prognosis of traumatic macular hole. Chinese J Gen Pract. (2012) 10:11-2.

44. Li XW, Lu N, Zhang L, Wang WW, Wang Y, Yan W, et al. Follow-up study of traumatic macular hole. Chinese J Ophthalmol. (2008) 44:786-9.

45. Jin XM, Yang JX Li JF. Traumatic macular hole and its spontaneous closure. Chinese J Ocular Trauma Occup Eye Dis. (2005) 27:4135. doi: 10.3760/cma.j.issn.2095-1477.2005.06.005

46. Yamashita T, Uemara A, Uchino E, Doi N, Ohba N. Spontaneous closure of traumatic macular hole. Am J Ophthalmol. (2002) 133:2305. doi: 10.1016/S0002-9394(01)01303-4

47. Wang X, Peng H. Treatment and research advances in therapy of traumatic macular hole. Recent Adv Ophthalmol. (2019) 39:583-8.

48. Mitamura Y, Saito W, Ishida M, Yamamoto S, Takeuchi S. Spontaneous closure of traumatic macular hole. Retina. (2001) 21:385-9. doi: 10.1097/00006982-200108000-00020

Conflict of Interest: The authors declare that the research was conducted in the absence of any commercial or financial relationships that could be construed as a potential conflict of interest.

Publisher's Note: All claims expressed in this article are solely those of the authors and do not necessarily represent those of their affiliated organizations, or those of the publisher, the editors and the reviewers. Any product that may be evaluated in this article, or claim that may be made by its manufacturer, is not guaranteed or endorsed by the publisher.

Copyright (c) 2021 Zhou, Feng, Lv, Fu, Xue and Ye. This is an open-access article distributed under the terms of the Creative Commons Attribution License (CC BY). The use, distribution or reproduction in other forums is permitted, provided the original author(s) and the copyright owner(s) are credited and that the original publication in this journal is cited, in accordance with accepted academic practice. No use, distribution or reproduction is permitted which does not comply with these terms. 